\title{
ICECUBE, THE WORLD'S LARGEST DARK MATTER DETECTOR
}

\author{
Hagar Landsman for the IceCube collaboration \\ Physics department, University of Wisconsin, \\ Madison, WI 53703, USA \\ *E-mail: hagar@icecube.wisc.edu \\ http://icecube.wisc.edu
}

\begin{abstract}
IceCube is a kilometer scale high-energy neutrino observatory, currently under construction at the South Pole. It is a photo-detector, using the deep Antarctic ice as detection medium for the Cherenkov photons induced by relativistic charged particles. These charged particles may be atmospheric muons or reaction products from neutrino interactions in the vicinity of the instrumented volume. The experiment searches for neutrinos originating in astrophysical sources, and can also detect neutrinos from WIMP interaction in the Sun or Earth. In the last two austral summers, 9 in-ice strings and 16 surface IceTop stations (out of up to 80 planned) were successfully deployed, and the detector has been taking data ever since. In this proceedings, IceCube design, present status, performance and dark matter detection sensitivities will be discussed.
\end{abstract}

Keywords: IceCube; Neutrino; AMANDA; WIMP; Neutralino; Earth; Sun

\section{Introduction}

High energy neutrino detectors consist of an array of light detectors ("optical modules") spread evenly in deep and clear media, like the Antarctic ice. They are designed to detect Cherenkov photons radiated by charged particles. The particles that are most likely to penetrate through the matter above the fiducial volume of the detector are muons (from interactions of cosmic rays in the atmosphere), and neutrinos (atmospheric or from any other source). Based on measurements of the number of photons arriving at different light detectors and their arrival times, a track or a cascade can be reconstructed.

The IceCube detector is a neutrino detector currently under construction at the South Pole. ${ }^{1}$ The main scientific goal of IceCube is to map the neutrino sky, searching for high energy neutrinos that may be cre- 
ated in hadronic processes, for example in Gamma-Ray Bursts or Active Galactic Nuclei. IceCube will also look for high energy GZK neutrinos ${ }^{2}$ that are expected to be created by interaction of the cosmic ray protons with the background radiation. It will study air showers, and high energy atmospheric neutrinos. ${ }^{3}$ In a special data acquisition mode, IceCube will detect supernovae neutrinos, where the detection probability depends on the distance and neutrino flux from the explosion. ${ }^{4}$ To some extent, IceCube measurements can be used for neutrino mass hierarchy and CP phase measurements. ${ }^{5}$

Any model predicting a certain neutrino flux, can be tested by IceCube. These sources include, but are not limited to, dark matter, super-symmetry, magnetic monopoles and quantum gravity.

The neutralino is the lightest Weakly Interacting Massive Particle (WIMP) candidate of the Minimal Super-Symmetric extension of the Standard Model (MSSM). It is stable due to R-parity conservation. In direct searches, the WIMPs are detected by the scattered nuclei in the rare elastic scattering of WIMPs on a suitable target. ${ }^{6}$ In indirect searches the annihilation products of two neutralinos are detected. If the decay products include neutrinos, neutrino telescopes like IceCube and AMANDA can be used for detection. ${ }^{7}$

Relic neutralinos from the Galactic halo can lose energy through scattering with matter and become trapped in the gravitational field of massive objects, such as the Sun and the Earth, where they accumulate over time and annihilate with each other. The decay products may vary, and most of them will interact and decay in the massive body. If neutrinos are created from those secondaries, they will escape and create a neutrino flux.

The number of detected neutrinos, from neutralino interaction, depends on the source characteristic (like capture rate, neutralino annihilation cross section and mass) and on the detector response.

\section{WIMPs searches in AMANDA}

AMANDA, the predecessor experiment to IceCube, consists of 677 optical modules deployed on 19 strings at depth between $1.5 \mathrm{~km}$ and $2 \mathrm{~km}$. A search for an excess of muon neutrinos from the center of the Earth or the Sun was preformed using the AMANDA detector. ${ }^{8,9}$

Muons created by cosmic-ray interactions in the atmosphere, can penterate through the 1-2 km of ice and trigger the detector. Their abundance will overpower smaller fluxes. They are filtered using the matter of Earth, blocking muons from the northen hemisphere, but allowing neutrinos to 
pass through. Therefore only events transversing through Earth, "upgoing events" are used. For neutrinos with energies above one PeV, the interaction cross section is high enough and Earth starts to become opaque, depending on the angle the neutrino comes in. For WIMP studies, we are intrested in lower energies since it is predicted that the mass of the neutralino will be less than $340 \mathrm{TeV}$, based on unitarity conditions.

Since the position of the Sun in the South Pole, reaches no more than $23^{\circ}$ below the horizon, it is not possible to exploit the full advantage of the upgoing events and the separation between signal and atmospheric muons is harder. In the case of searches for WIMPs from the center of the Earth, the expected signal is veritically upgoing, and the atmospheric muon background is easily removed, with no signal loss.

In both cases, tracks are reconstructed using times of hits in optical modules at known positions in the ice. The reconstructed zenith angle is used to separate background events from signal in the Earth analysis, and the space angle between the Sun and the reconstructed track is used to separate signal from background for the Sun analysis.

The neutralino induced events were simulated using the DARKSUSY ${ }^{10}$ program, with neutralino masses in the range of $100 \mathrm{GeV}$ to $5 \mathrm{TeV}$. Two decay channels were analyzed: A 'soft' channel where the neutralinos annihilate to a pair of b-quarks, and a 'hard' channel where they decay to W-bosons.

The background consists of atmospheric neutrinos that arrive to the detector from all directions (upgoing and downgoing events) and from mis-reconstructed downgoing muon. The background is estimated using off source measurements and simulation. No excess of signal was observed in either the Sun or the Earth analysis.

Based on theoretical models and simulation this result is translated to upper limits on the flux of neutrinos originating from the Sun or from the Earth, and further extrapolated to limits on models. These predictions are model dependent. Not only they depend on SUSY models, that determine branching ratios, but to some extent also on astrophysical assumptions. The threshold energy of the detector plays an important role in the limit extrapolation. As can be seen in figures 1 and 2, once completed, based on the current simulations, IceCube will contribute to the limits of WIMPs from the Sun. 


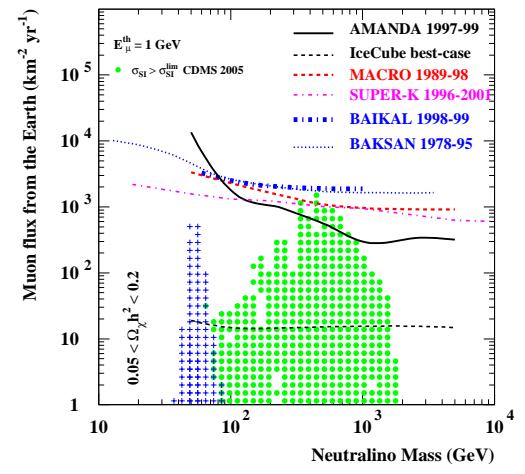

Fig. 1. Upper limits on the muon flux from the Earth, from neutralino annihilation in the 'hard' channel, as a function of the neutralino mass. IceCube best case refers to simulation of 80 strings with 3 years worth of data.

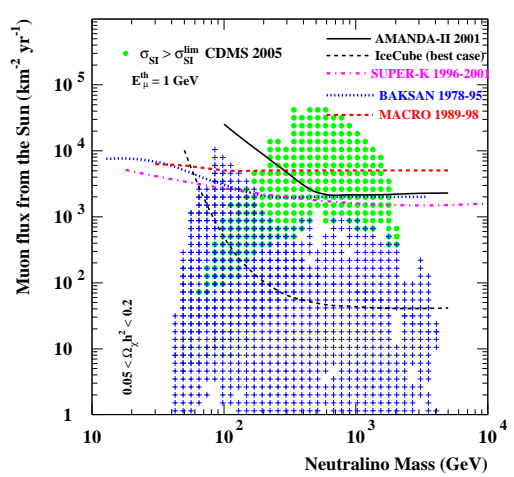

Fig. 2. Upper limits on the muon flux from the Sun, from neutralino annihilation in the 'hard' channel, as a function of the neutralino mass. The crosses show predictions based on DARKSUSY. The dots show models disfavored by direct searches. The limits were rescaled to muon threshold of $1 \mathrm{GeV}$.

\section{Current Status and Verification of IceCube}

A larger and improved detector will increase the number of events that can be measured and improve the track reconstruction resolution allowing better separation between signal and background. This will increase the sensitivity for WIMPs, and allow us to measure more neutralinos, in case of detection, or set better limits on models.

The IceCube detector will consist of up to 4800 Digital Optical Modules (DOMs) covering a fiducial volume of 1 cubic km. ${ }^{1}$ The DOMs will be equally spaced on up to 80 strings at depths from 1.5 to $2.5 \mathrm{~km}$ in the clear Antarctic ice. An array of surface stations, called IceTop, enhances the ability to trigger on, or veto, down-going showers. Each IceTop station consists of two clear ice tanks, each instrumented with 2 DOMs. An IceTop station is located roughly 10 meters from each bore hole of the array.

Each DOM is an autonomous data collecting and digitizing unit consisting of a 10" Hamamatsu PMT in a 13" pressure sphere. A main board inside the DOM can digitize up to 300 Mega Samples per Second (MSPS) for $400 \mathrm{~ns}$ and 40 MSPS for $6.4 \mu \mathrm{s}$. A flasher board, equipped with 12 LEDs, produces pulses used for optical and timing calibration.

In the winter of 2004-2005 a single IceCube In-Ice string and 4 IceTop 
stations were deployed. At the end of the 2006 Austral summer IceCube consisted of 9 strings and 16 IceTop stations. A set of measurements was performed after the first year to confirm the design goal of the detector and check its performance. ${ }^{11}$

\subsection{Timing}

In order to reconstruct tracks over the entire array, a timing resolution of a few nanoseconds is needed. The time calibration of the entire array is done by sending a fast bipolar pulse at known intervals from the surface to every DOM. Each DOM then sends an identical fast bipolar pulse back to the surface after a known delay. Based on the time measured on the surface and on the DOM, and based on the shape of the measured pulse, the round trip time of the signal is calculated. Figure 3 shows the round trip times of the calibration pulse. The time increases with the depth of a DOM, and is constant for the IceTop array. The variation of the round trip time from one calibration to the next provides a measurement of the time calibration accuracy (figure 4).

The time resolution of each DOM was verified in two independent ways. First, a LED on a DOM was flashed and an adjacent DOMs triggered on it. The time delay between the flashing and the triggering was measured multiple times. This procedure was repeated for all DOMs, and the maximum time RMS resolution was found to be less than $2 \mathrm{~ns}$. A different way to estimate the time resolution is by reconstructing down-going muon tracks excluding one DOM, and calculating the time difference between the measured hit time and the expected hit time. Figure 5 shows the distribution of those time residuals for a single DOM using multiple events. The process is repeated for all DOMs. The resolution was found to be less than $3 \mathrm{~ns}$, after accounting for ice properties.

\subsection{Muon track reconstruction}

In order to point back to the origin of the neutrinos, a muon track is reconstructed and the zenith and azimuth angles are calculated. Reconstruction of muon tracks, originating from downward going cosmic rays, or from neutrino interactions in the detector volume, is done by analyzing the waveform measured on each DOM.

A comparison of the measured atmospheric neutrinos and downgoing muons flux to the predicted and known fluxes is an important consistency check on the detector response and simulation. The quality of the recon- 


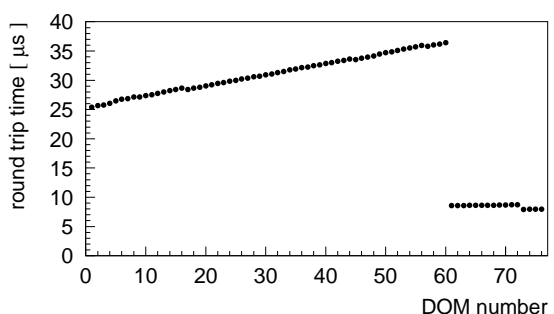

Fig. 3. Round trip time of the time calibration pulse. DOM number 1 is at the top of the string, and DOM 60 is at the bottom. DOMs 60-76 are part of the surface array.

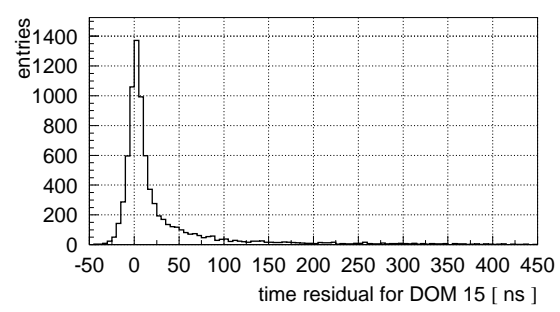

Fig. 5. Distribution of time residuals of photons arriving at a DOM from nearby tracks reconstructed with the rest of the string.

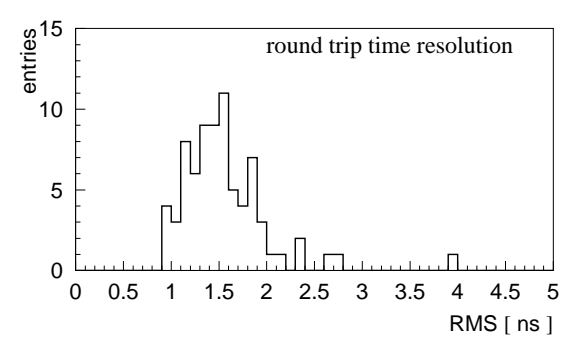

Fig. 4. The rms variation of the round trip time of the time calibration pulse for 76 DOMs on IceCube's first string and 4 IceTop tanks.

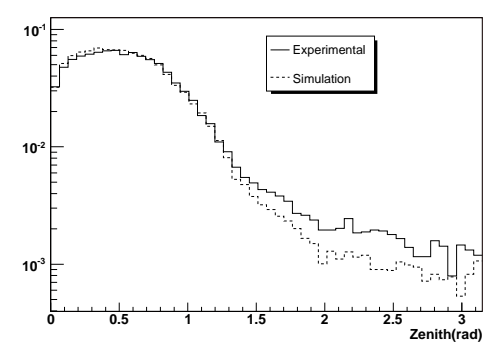

Fig. 6. Distribution of reconstructed and measured zenith angle for downgoing events.

struction depends on the number of DOMs hit (determined by the geometry and the energy of the event), the ice quality (impurities lead to photon scattering and absorption) and timing resolution. Figure 6 compares the measured downgoing events reconstructed zenith angle to the predicted one using simulation of downgoing muons. Figure 7 shows the predicted angular resolution of the IceCube detector with 9 and 80 strings.

\subsection{Summary}

A neutrino flux from the center of the Earth or the Sun, may indicate neutralino capture inside those heavy bodies. No excess of events from the Sun or the Earth, above the estimated atmospheric neutrino background, was measured by the AMANDA detector. Limits on the fluxes have been set for different neutralino masses.

The IceCube detector, under construction in the South Pole will be 


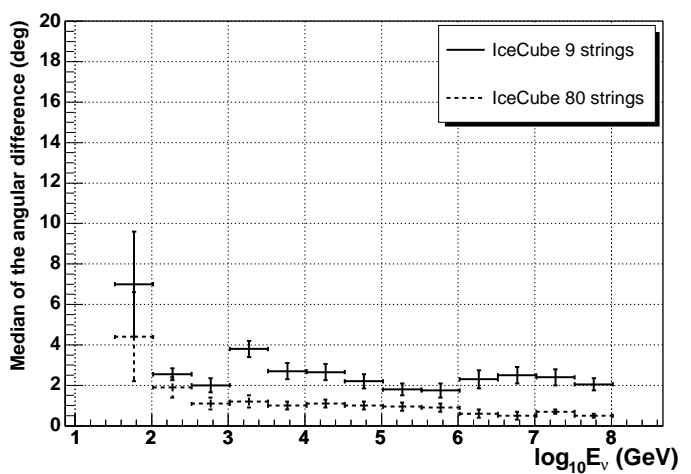

Fig. 7. The angular resolution for 9 strings and for the full IceCube array as function of event energy (preliminary).

a powerful tool for high energy neutrino searches. Among other results, it will also improve the limits on neutrinos from neutralino annihilation, in the case of no signal. The higher threshold energy will decrease its sensitivity to lower mass neutralinos, but its larger size and better track reconstruction abilities, together with data collected on AMANDA, will improve the limits, especially for higher mass. Until December 2007, IceCube consisted of 9 strings and 16 surface stations. The detector will rapidly grow as more strings and stations will be deployed. Measurements done on IceCube show good timing and reconstruction ability throughout the detector.

\section{References}

1. IceCube Project Preliminary Design Document, Ahrens et al. (IceCube Collaboration), http://icecube.wisc.edu.

2. R.Engel, D.Seckel and T.Stanev, Phys.Rev. D64 093010 (2001)

3. M.C. Gonzalez-Garcia, F.Halzen and M.Maltoni, Phys.Rev. D71, 092010 (2005)

4. J.Ahrens et al. (AMANDA Collaboration), Astropart. Phys. 16 , 345 (2002)

5. W. Winter, Phys.Rev. D74, 033015 (2006)

6. CDMS Collaboration, Phys.Rev.Lett. 93211301 (2004)

7. F. Halzen and D.Hooper, Phys. Rev. D73 1233507 (2006)

8. A. Achterberg et al. (IceCube collaboration), Astropart. phys. 24 (2006) 459466

9. A. Achterberg et al. (IceCube collaboration), Astropart. Phys. 26 (2006) 129139

10. P.Gondolo et al., Journ. of Cosm. \& Astropart. Phys. 0407, 008, 2004

11. A. Achterberg et al. (IceCube collaboration), Astropar. Phys. 26, (2006) 155 